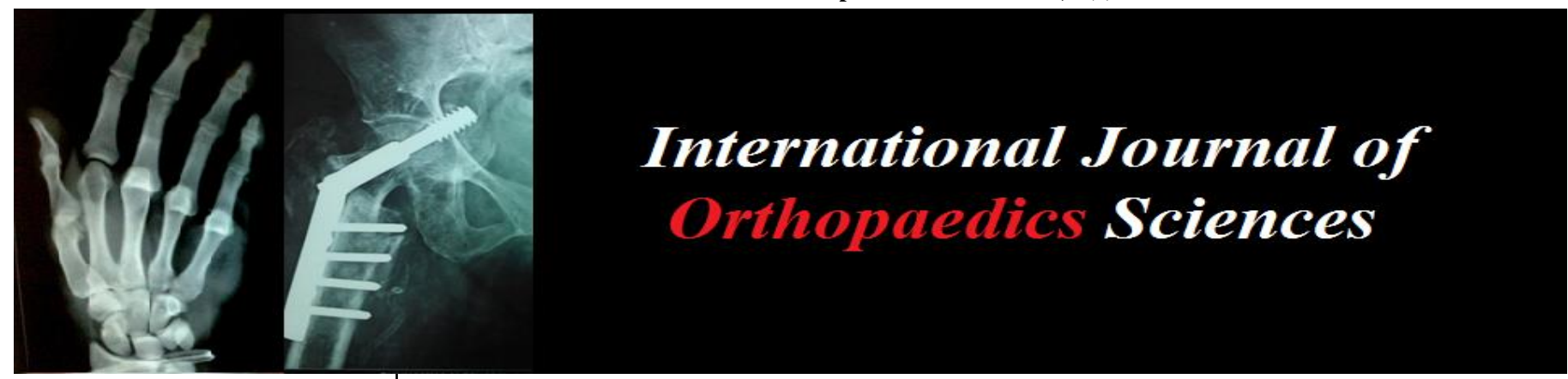

E-ISSN: 2395-1958

P-ISSN: 2706-6630

IJOS 2020; 6(1): 537-539

(C) 2020 IJOS

www.orthopaper.com

Received: 04-11-2019

Accepted: 08-12-2019

Dr. Mohan Babu L

DNB (Ortho), Assistant

Professor, Birrd (T) Hospital.

Tirupathi, Andhra Pradesh,

India
Corresponding Author: Dr. Mohan Babu L

DNB (Ortho), Assistant Professor, Birrd (T) Hospital. Tirupathi, Andhra Pradesh, India

\section{Component asymmetry evaluation in 200 bilateral total knee arthroplasty cases}

\section{Dr. Mohan Babu L}

DOI: https://doi.org/10.22271/ortho.2020.v6.i1j.1918

\section{Abstract}

Background: The importance of correct sizing of components for TKA for an optimal function and long-term results has been stressed in many reports. Proper sizing of both femoral and tibial component in both knees is necessary to maximize outcomes and reduce the risk of complications.

Aims: To analyse the incidence of asymmetry of femoral and tibial components among bilateral knee Arthroplasty cases.

Methods: A retrospective review of operative notes and outpatient charts was performed on all patients undergoing simultaneous and two-staged bilateral TKA at this institution from 2014 to 2016.

Conclusion: Good range of movement of both knee joints provides the support that proper component selection whether symmetrical or asymmetrical is the keystone for successful bilateral total knee arthroplasty.

Keywords: Component asymmetry, evaluation, arthroplasty cases

\section{Introduction}

Total knee arthroplasty is the best surgical procedure indicated in the last stage of osteoarthritis, rheumatoid arthritis, posttraumatic arthritis to provide pain relief.

Many factors play a role in the outcome of TKA, out of which the final proper sizing of the components also plays an important role. The importance of correct sizing of components for TKA for an optimal function and long-term results has been stressed in many reports. Proper sizing of both femoral and tibial component in both knees is necessary to maximize outcomes and reduce the risk of complications ${ }^{[1]}$.

Notching of the anterior cortex decreases axial and torsional load to failure and increases the risk of periprosthetic femoral fractures.

To prevent notching, a surgeon may overstuff the patellofemoral joint, Overstuffing may result in extensor mechanism tightness and subsequent reduction in postoperative knee flexion, but the amount of overstuffing that might decrease postoperative flexion.

Improper component sizing may adversely affect functional results including range of motion (ROM) and stability.

So the size of the femoral component is key for preventing above mentioned mechanisms.

Oversizing tibia components lead to excessive lateral pressure which leads to pain, undersizing increases spacer wear and tear ${ }^{[2]}$. To prevent above-mentioned complications we should use the appropriate size of the tibial component ${ }^{[3]}$

In the current study, we did a retrospective analysis of all the patients who underwent bilateral TKA with the hypothesis that both sides in these patients may not be symmetrical in all. We analyzed the incidence of asymmetry of femoral and tibial components among bilateral knee Arthroplasty case. The purpose of this study was to quantify the incidence of femoral and tibial, size asymmetry in simultaneous bilateral TKA.

\section{Material and Methods}

A retrospective review of operative notes and outpatient charts was performed on all patients undergoing simultaneous and two stages of bilateral TKA at this institution from 2014 to 2016. This study underwent evaluation and received approval by the ethical committee

$$
\sim 537 \sim
$$


We included cases

a. Where prostheses of the same manufacturer were used in both the knees.

b. Who had data regarding the implant details available?

c. Who had a minimal follow-up of 1 year?

Cases excluded were

a. Patients whose follow up records were lost.

b. Those who had any postoperative complications like infection.

c. Revision total knee replacement cases.

Detailed history, clinical assessment, anteroposterior and lateral x-rays and routine blood investigations were done. A total of 200 patients were studied. They are divided into Group A and Group B

Group A: Includes where both knees total knee arthroplasty was done in a single sitting.

Group B: Includes cases where 6 months' time interval was taken to operate between two knees.

Group A was operated by a single surgeon; Group B is operated by two different surgeons.

All cases cruciate-retaining arthroplasty was done. The implants used included Optetrak-CR Extech. The groups were analyzed for the component asymmetry between the two knees. All the comparative analysis was done between those cases which had component asymmetry and those where the bilateral components were symmetrical, individually in both the groups. All the statistical analysis was done using SPSS 12.0 software. For all purposes, a $P$-value $<0.05$ was considered to be statistically significant.

\section{Results}

The diagnosis was primary osteoarthrosis in 200 cases and. There were 100cases where arthroplasty was done on the same day (group A) and 100 cases where a staged arthroplasty was done at 6 months interval (group B).

The mean age of the patients in group A was $64.7+6.54$ years while it was $64.7+$ years $(65-82$ yrs) in group B. The male-tofemale ratio was 44:56 in group $A$, while it was 50:50 in group B.

The femoral asymmetry occurred in a total of 2 cases $(2 \%)$, while the tibial component asymmetry seen in 5 cases $(5 \%)$ in group A, whereas group B femoral asymmetry is seen in $4(4 \%)$, tibial asymmetry in 8 cases $(8 \%)$. The male-female difference in these cases was found to be insignificant for both the femoral (P-value 0.36$)$ and tibial component $(P$ value 0.35$)$.

Table: They are divided into Group A and Group B

\begin{tabular}{|c|c|c|}
\hline Group & Femoral asymmetry & Tibial asymmetry \\
\hline Group A & $2 \%$ & $5 \%$ \\
\hline Group B & $4 \%$ & $8 \%$ \\
\hline
\end{tabular}

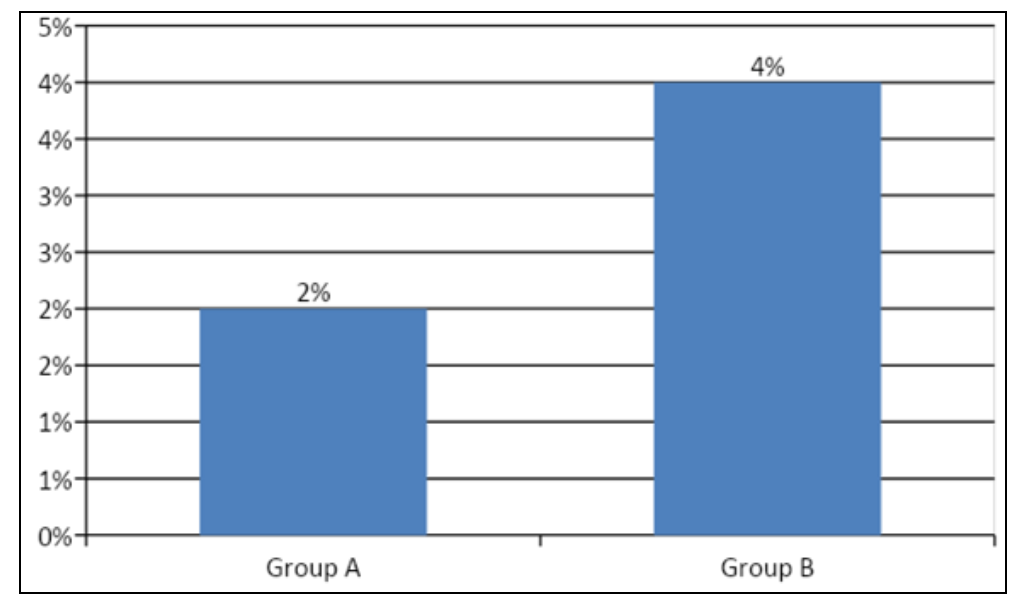

Fig 1: Fig show of different in group A and group B

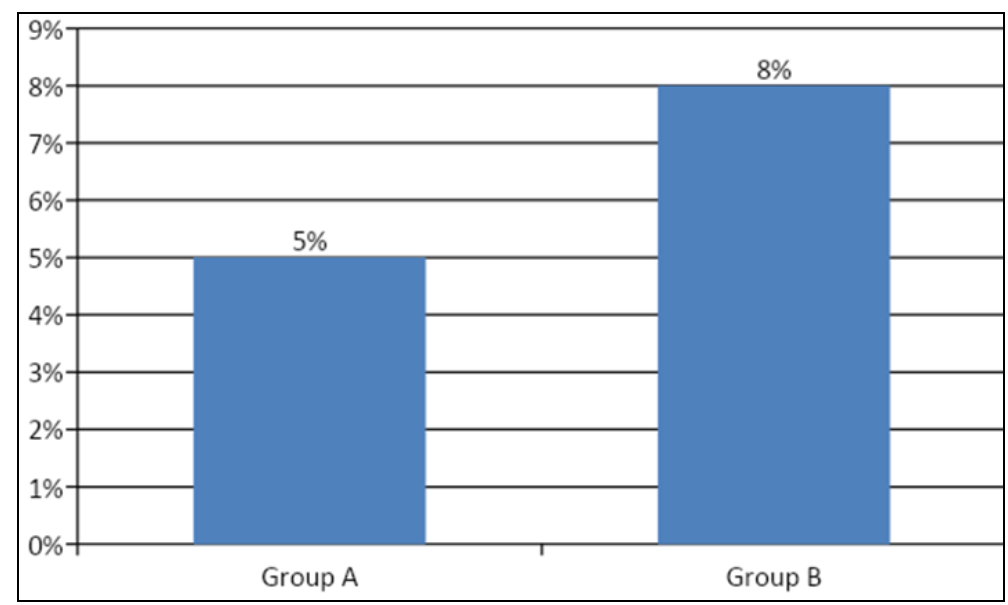

Fig 2: Fig show of different in group A and group B 
The difference was statistically insignificant ( $P$ value 0.40$)$. Of the 6 cases of femoral asymmetry, in 5 cases the asymmetry was by 1 size, while in 1 case it was 2 sizes apart.

In cases of tibial asymmetry, 13 cases were 1 size apart while in 4 cases it was 2 sizes apart.

The mean preoperative ROM in group A was 105.09+/- 7.02 while in group B it was $110.00+/-7.38$.

The mean preoperative NOKS in group A was 15 , in group B $14.67+/-5.146$ Postoperative ROM of group A $120.32+3.0$, in group B 122.58, post-operative NOKS IN group A 41.89 +1.67 , in group B $41.46+2.264$ and postoperative NOKS. Statistical analysis showed no significant difference between these cases in both groups.

Though there is a great improvement in preoperative to postoperative status, there no significant difference in postoperative scores.

\section{Discussion}

Variations in the size of normal knee anatomy have been analysed, but asymmetryduringarthroplastynot emphasised ${ }^{[5]}$. In this study, both surgeons while doing the bilateral TKAs independently sized components for each knee during the arthroplasty observed a difference between right and left knees in terms of bone anatomy in a good number of patients. Whether the bilateral TKA was symmetrical or asymmetrical, the postoperative knee scores and ROM provide the support that proper component selection was achieved for each individual knee. The pitfall of this study is that the radiological staging of the disease and severity of the disease is not considered as this is a retrospective study and true anthropometric measurements of the femur and tibia were not done either pre or per operatively, which would better document the actual difference of sizes in these knees.

Brown and Diduch reported asymmetry rates for femoral $(6.7 \%)$, tibial $(1.1 \%)$, and patellar components $(0.3 \%)$ in a review of 268 patients who underwent either simultaneous or staged bilateral TKA ${ }^{[2]}$. Capeci et al. reported the asymmetry rates of $8.7 \%, 6.7 \%$, and $5.1 \%$ for femoral, tibial, and patellar components, respectively in a review of 253 patients who underwent simultaneous bilateral TKA ${ }^{[4]}$, In Mootha et al. ${ }^{[6]}$. the study, they found a higher incidence of $9.3 \%$ for the femoral component and $8.6 \%$ for the tibial component compared to Capeci et al.

In our study, out of 200 cases, we found asymmetry rate of $6 \%$ in femoral components and $13 \%$ asymmetry in tibial components.

For good function and maximum stability of joint, proper sizing of components is essential. Flexion extension gap disparity can occur when incorrectly sized femoral component is used. Tight flexion gap occurs when oversized femoral component is used. Owing to this patellar instability, anterior knee pain and decreased range of movement due to tight flexion gap. Flexion instability will occur if the undersized femoral component is used. Oversized tibial component causes lateral overhanging and pain induced impingement of popliteal tendon or IT band. The undersized tibial component will cause sinking of the implant in osteoporotic patients.

While doing bilateral total knee replacement, relying on the opposite knee sizes will lead to improper component selection. Knowledge of component asymmetry helps in achieving good results in bilateral TKA's. Bony anatomical differences in size between the right and left knees were identified in a good number of patients when bilateral TKA was done in a single sitting by two different surgeons. Good range of movement of both knee joints provides the support that proper component selection whether symmetrical or asymmetrical is the keystone for successful bilateral total knee arthroplasty.

\section{References}

1. Mihalko W, Fishkin Z, Krackow K. Patellofemoral overstuff and its relationship to flexion after total knee arthroplasty. Clin Orthop Relat Res. 2006; 449:283-7.

2. Brown TE, Diduch DR, Moskal JT. Component size asymmetry in bilateral total knee arthroplasty. Am J Knee Surg. 2001; 14:81.

3. Insall JN, Easley ME. Surgical techniques and instrumentation in total knee arthroplasty. In: Insall JN, Scott WN, editors. Surgery of the knee. Philadelphia, PA: Churchill Livingstone, 2001, 1553-1620.

4. Capeci CM, Brown Ec, Scuderi GR, Scott NW. Component asymmetry in total knee arthroplasty. J Arthroplasty. 2006; 21:749-53.

5. Chin KR, Dalury DF, Zurakowski D, Scott RD. Intraoperative measurements of male and female distal femurs during primary total knee arthroplasty. J Knee Surg. 2002; 15:213.

6. Reddy VG, Mootha AK, Thai C, Kantesaria P, Kumar RV, Reddy D. Are both the knees of the same size? Analysis of component asymmetry in 289 bilateral knee arthroplasty. Indian J Orthop. 2011; 45:251-4. 